\title{
Telomere dysfunction in alveolar epithelial cells causes lung remodeling and fibrosis
}

\author{
Ram P. Naikawadi, ${ }^{1}$ Supparerk Disayabutr, ${ }^{1}$ Benat Mallavia, ${ }^{1}$ Matthew L. Donne, ${ }^{2}$ Gary Green, ${ }^{1}$ \\ Janet L. La, ${ }^{1}$ Jason R. Rock, ${ }^{2}$ Mark R. Looney, ${ }^{1}$ and Paul J. Wolters ${ }^{1}$ \\ 'Division of Pulmonary, Critical Care, Allergy and Sleep Medicine, Department of Medicine, University of California, \\ San Francisco, California, USA. ²Department of Anatomy, School of Medicine, University of California, San Francisco, \\ California, USA.
}

\begin{abstract}
Telomeres are short in type II alveolar epithelial cells (AECs) of patients with idiopathic pulmonary fibrosis (IPF). Whether dysfunctional telomeres contribute directly to development of lung fibrosis remains unknown. The objective of this study was to investigate whether telomere dysfunction in type II AECs, mediated by deletion of the telomere shelterin protein TRF1, leads to pulmonary fibrosis in mice (SPC-Cre TRF T//7/ mice). Deletion of TRF1 in type II AECs for 2 weeks increased $\gamma \mathrm{H} 2 \mathrm{AX}$ DNA damage foci, but not histopathologic changes in the lung. Deletion of TRF1 in type II AECs for up to 9 months resulted in short telomeres and lung remodeling characterized by increased numbers of type II AECs, $\alpha$-smooth muscle actin ${ }^{+}$mesenchymal cells, collagen deposition, and accumulation of senescence-associated $\beta$-galactosidase ${ }^{+}$lung epithelial cells. Deletion of TRF1 in collagen-expressing cells caused pulmonary edema, but not fibrosis. These results demonstrate that prolonged telomere dysfunction in type II AECs, but not collagen-expressing cells, leads to age-dependent lung remodeling and fibrosis. We conclude that telomere dysfunction in type II AECs is sufficient to cause lung fibrosis, and may be a dominant molecular defect causing IPF. SPC-Cre TRFT//f mice will be useful for assessing cellular and molecular mechanisms of lung fibrosis mediated by telomere dysfunction.
\end{abstract}

Conflict of interest: The authors declare that no conflict of interest exists.

Submitted: January 25, 2016 Accepted: August 4, 2016 Published: September 8, 2016

Reference information: JCI Insight. 2016;1(14):e86704. doi:10.1172/ji.insight.86704.

\section{Introduction}

Idiopathic pulmonary fibrosis (IPF) is a terminal, age-associated, interstitial lung disease (ILD) with an overall prevalence of 14 to 43 per 100,000 people (1). Consistent with the association with aging, telomere dysfunction has been implicated in the pathogenesis of $\operatorname{IPF}(2,3)$. Evidence includes the observation that telomeres are short in type II alveolar epithelial cells (AECs) of IPF patients $(4,5)$, that type II AEC expression of senescence markers is unique to IPF lungs (6), and that peripheral blood leukocyte telomere length predicts survival in IPF patients (7). Variants in genes regulating telomere length including telomerase reverse transcriptase (TERT), RNA component of telomerase (TERC), and oligosaccharide-binding fold containing 1 (OBFC1) genes have been identified as susceptibility loci for development of sporadic IPF (8). Similarly, mutations in TERT, TERC, regulator of telomere elongation helicase 1 (RTEL1), and poly(A)-specific ribonuclease $(P A R N)$, have been identified in kindreds of familial pulmonary fibrosis (4, 9-11). Pulmonary fibrosis is also found in a subset of patients with dyskeratosis congenita (DKC), a disease of telomere dysfunction $(12,13)$. Although these studies implicate telomere dysfunction in the development of pulmonary fibrosis, evidence that telomere dysfunction contributes directly to the development of lung fibrosis is lacking.

Telomeres consist of tandem TTAGGG repeats and associated proteins (shelterin complex) that form a capping structure at the ends of chromosomes, which protects them from degradation and activation of DNA repair responses (14). Telomeres shorten with each cell division. When critically shortened, cells senesce due to uncapping of telomeres and activation of cell-cycle arrest programming. Modeling the biological consequences of telomere shortening in mice has been limited because murine telomeres are 5-10 times longer and their lifespan 30 times shorter than those in humans (15). One approach for studying telomere biology in vivo has been to conditionally delete proteins of the shelterin complex, leading to the uncapping of telomeres. When shelterin complex proteins are deleted from specific cell types, phenotypes consistent with dysfunctional telomeres manifest (16-18). The experiments 
A
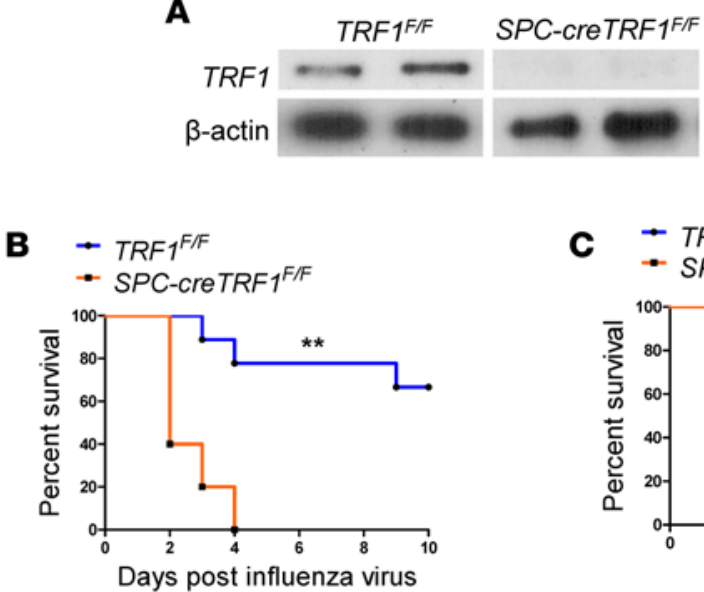

D

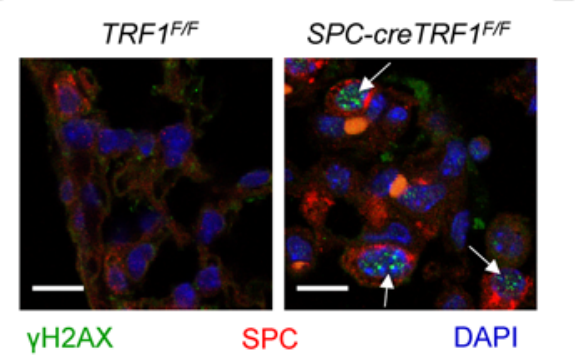

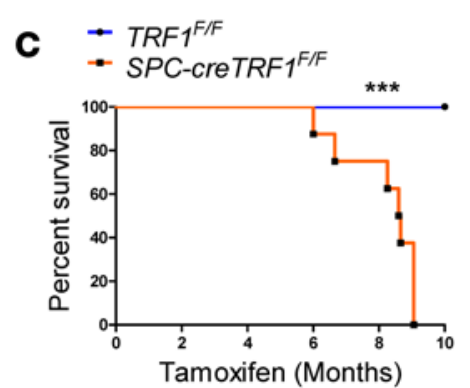

$\mathbf{E}$

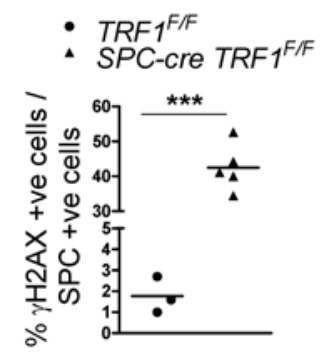

Figure 1. Telomere dysfunction in type II AECs results in DNA damage and increased susceptibility to influenza virus, spontaneous lung remodeling, and death. (A) Immunoblot showing TRF1 deletion in type II AECs isolated from tamoxifen-treated $T R F 7^{f / f l}$ and $S P C$-Cre $T R F^{f / / f l}$ mice. (B) Survival of $T R F 7^{f / f l}$ and SPC-Cre TRF $7^{f / f l}$ mice following influenza virus infection $(A / P R / 8 / 34)$. Mice were treated with tamoxifen ( $250 \mathrm{mg} / \mathrm{kg}$ body weight) weekly for 4 weeks prior to infection. $n=10$ mice/group, ${ }^{* *} P=0.001$, log-rank test. (C) Survival of $T R F 7^{f / f l}$ and $S P C-C r e T R F 7^{f / f l}$ mice treated with weekly injections of tamoxifen $(250 \mathrm{mg} / \mathrm{kg}$ body weight). $n=10$ mice per group, ${ }^{* *} P<0.0001$, log-rank test. (D) $\gamma \mathrm{H} 2 \mathrm{AX}$ immunostaining of lung sections from $\mathrm{TRF}^{\text {fl/fl }}$ and SPC-Cre TRF/f/f mice treated with tamoxifen $(250 \mathrm{mg} /$ $\mathrm{kg}$ body weight) for 2 weeks. Note the $\gamma \mathrm{H} 2 \mathrm{AX}$ foci (green immunofluorescence, white arrows) in the nuclei of SPC-Cre $T R F T^{f / f l}$ mice. SPC, surfactant protein C (red immunostain). Nuclei were stained with DAPI (blue). Scale bars: $10 \mu \mathrm{m}$. (E) Quantification of percentage of $\gamma \mathrm{H} 2 \mathrm{AX}$-positive nuclei in SPC-positive cells. $n=3-5$ mice/group, ${ }^{* *} P=0.0002$, 2-tailed Student's $t$ test.

reported in this study adopt these approaches by conditionally deleting telomere repeat binding factor 1 (TRF1) in type II AECs or collagen-expressing cells to examine the consequence of dysfunctional telomeres in resident lung cells.

\section{Results}

Telomere dysfunction in AECs. To examine the consequences of type II AEC-specific telomere dysfunction, we adapted approaches used to study telomere dysfunction in keratinocytes and leukocytes $(16,17)$ and crossed $T R F 1^{f l f l}$ mice with mice capable of inducibly expressing Cre recombinase in surfactant protein Cexpressing cells (SPC-Cre ER ${ }^{T 2}$ rtTA mice, which contain tamoxifen-inducible Cre-modified estrogen receptor fusion protein) to generate $S P C$-Cre $T R F 1^{f / f l}$ mice (19). Following intraperitoneal tamoxifen injections, deletion of TRF1 was confirmed by immunoblot in type II AECs isolated from SPC-Cre TRF1 $1^{f l f l}$ mice (Figure 1A). Lungs of $S P C$-Cre $T R F^{1 / f / f}$ mice treated with weekly tamoxifen injections for 2 weeks were histopathologically normal (Figure 2, B and F, and Supplemental Figure 1B; supplemental material available online with this article; doi:10.1172/jci.insight.86704DS1) but had higher mortality following intranasal infection with influenza virus (A/PR/8/34) compared with tamoxifen-treated $T R F 1^{f l f l}$ mice (Figure $1 \mathrm{~B}$ ), indicating worse inflammatory lung injury in mice with type II AEC telomere dysfunction. At this time point, in the absence of influenza viral infection, lungs of SPC-Cre TRF1 $1^{f / f l}$ mice had increased $\gamma \mathrm{H} 2 \mathrm{AX}$ foci in type II AECs (Figure 1D), consistent with the uncapping of telomeres in these cells. Quantification revealed that $45 \%$ of total SPC-positive cells were also $\gamma \mathrm{H} 2 \mathrm{AX}$ positive (Figure 1E).

Deletion of TRF1 in lung epithelial cells leads to the development of lung fibrosis. When mice were treated with weekly injections of tamoxifen for up to 9 months, it was found that SPC-Cre TRF1 $1^{f / f l}$ mice have worse survival than tamoxifen-treated $T R F 1^{f / f l}$ control mice. Mice began to die 6 months after tamoxifen administration and no mice survived longer than 9 months of weekly tamoxifen treatments (Figure 1C). To determine why SPC-Cre TRF $1^{f l / f l}$ mice had impaired survival, the lungs of SPC-Cre TRF1 $1^{f l / f l}$ mice and $T R F 1^{f l f l}$ control mice were studied following 3 months of weekly tamoxifen injections or when moribund (following 6-9 months of weekly tamoxifen injections). SPC-Cre TRF flffl $^{f / c e}$ challenged with tamoxifen for 3 months showed subtle areas of lung remodeling, with scattered areas of septal thickening (Figure 2, C and G, and Supplemental Figure 1C). In contrast, SPC-Cre TRF1 fl/fl mice that 


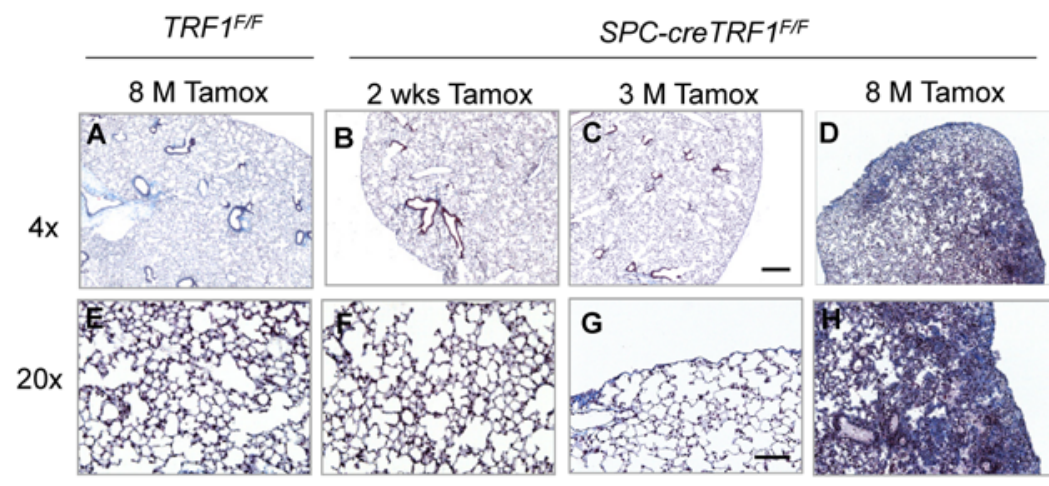

I

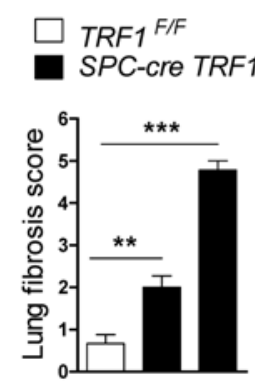

Tamox 8M 3M 8M
$J$

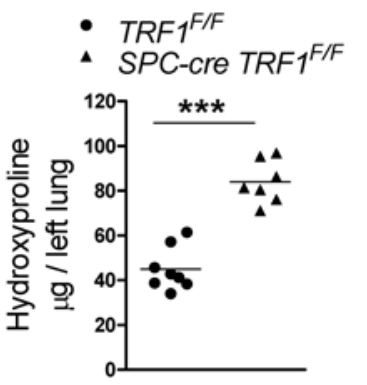

K

- $T R F 1^{F / F}$

- SPC-CreTRF1 ${ }^{F / F}$

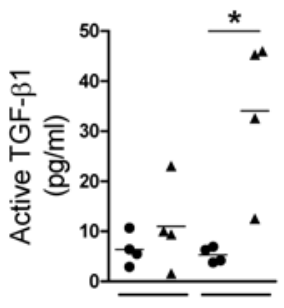

Tamox $3 \mathrm{M} \quad 8 \mathrm{M}$
Figure 2. Telomere dysfunction in type II AECs results in lung remodeling and death. (A-H) Masson's trichrome-stained lungs harvested after 2 weeks ( 2 wks), 3 months ( $3 \mathrm{M}$ ) and 8 months ( $8 \mathrm{M}$ ) of weekly injections of tamoxifen. Scale bars: $400 \mu \mathrm{m}$ ( $\times 4$ magnification) and $100 \mu \mathrm{m}$ ( $\times 20$ magnification). (I) Scoring

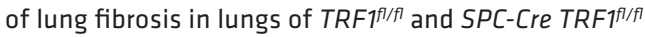
mice based on Ashcroft scale. $n=6-9$ mice per group, ${ }^{*} P<0.01,{ }^{* * *} P<0.0001,1$-way ANOVA. (J) Quantification of hydroxyproline in lungs of $T R F 7^{f / f l}$ and $S P C$-Cre TRF $7^{f / f l}$ mice harvested following treatment for 8 months with weekly injections of tamoxifen. $n=7-8$ mice/group, ${ }^{* *} P<0.0001,2$-tailed Student's $t$ test. (K) Active TCF- $\beta 1$ was measured by ELISA in bronchoalveolar fluid samples obtained from $T R F 7^{f / f l}$

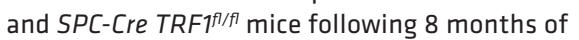
weekly tamoxifen injections. $n=4$ mice/group ${ }^{*} P<0.05,2$-tailed Student's $t$ test.

received tamoxifen injections for 6-9 months were found to have alveolar septal thickening, architectural distortion, and deposition of interstitial collagen (Figure 2, D and H, and Supplemental Figure 1D) compared with age-matched, tamoxifen-treated, TRF1flfl control mice (Figure 2, A and E, and Supplemental Figure 1A). When extent of lung fibrosis was scored in a blinded manner using the Ashcroft scale (20), the $S P C$-Cre $T R F 1^{f / f l}$ mice receiving tamoxifen for 8 months had a significantly higher score compared with $S P C$-Cre $T R F 1^{f l f l}$ mice treated with tamoxifen for 3 months or TRF1 $1^{f / f l}$ controls (Figure 2I). Furthermore, the levels of hydroxyproline (Figure 2J) and active TGF- $\beta 1$ (Figure 2K), were elevated in lungs of terminal SPC-Cre TRF1 $1^{f l f l}$ mice compared with $T R F 1^{f / f l}$ control lungs. Analysis of bronchoalveolar lavage (BAL) fluid cell counts and differentials revealed increased numbers of mononuclear cells (Figure 3A) and slight increases in neutrophils (Figure 3B) and lymphocytes (Figure 3C). Immunophenotyping of lung homogenate revealed no difference in the relative fractions of leukocyte subtypes including B cells, T cells, Th17 cells, fibrocytes, and CD206 ${ }^{+}$macrophages (Supplemental Table 1) in SPC-Cre TRF1 $1^{f / f l}$ mice compared with $T R F 1^{f / f l}$ control lungs. These findings show that isolated deletion of TRF1 in type II AECs leads to the spontaneous development of lung fibrosis and that this fibrosis may be mediated, in part, by TGF- $\beta 1$.

Figure 3. Bronchoalveolar lavage fluid (BALF)

analysis. (A-C) Cell count and differentials of BALF

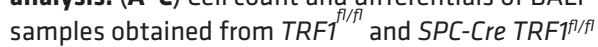
mice after 8 months of weekly tamoxifen injections. $n=4$ mice/group, ${ }^{*} P<0.05$, 2-tailed Student's $t$ test. (A) Macrophages, (B) PMNs, and (C) Lymphocytes.
A

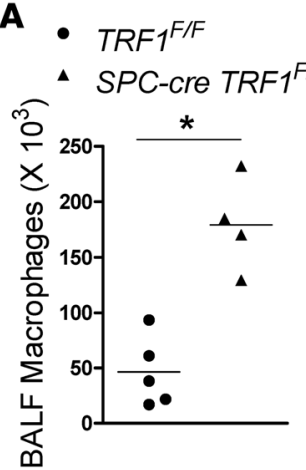

B - SPC-cre TRF1F/F

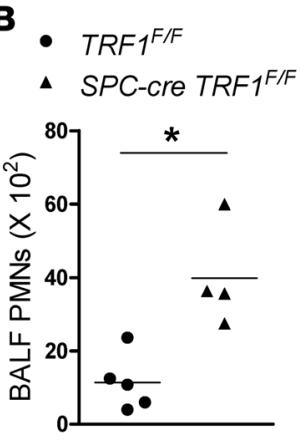

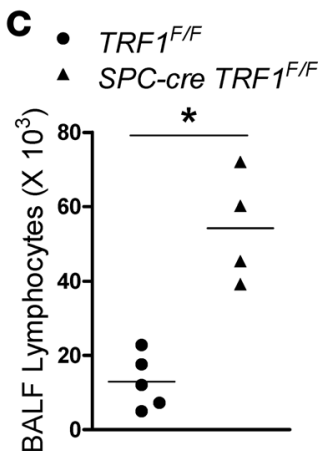


A

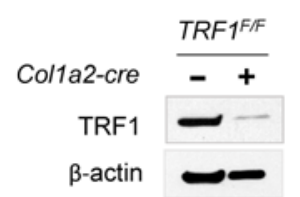

B $-T R F 1^{F / F}$

- Col1a2-cretRf1 ${ }^{\mathrm{F} / F}$

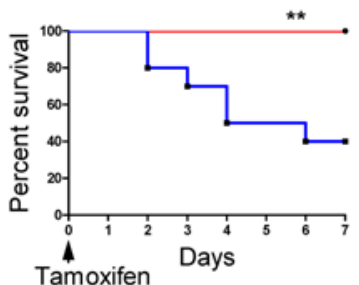

Tamoxifen
TRF1FIF Col1a2-creTRF1F/F

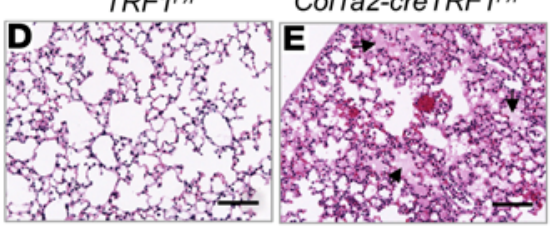

H

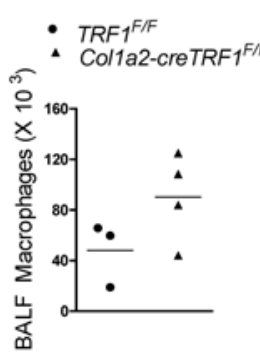

I

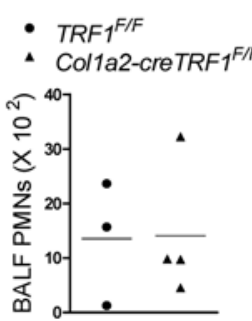

TRF1F/F

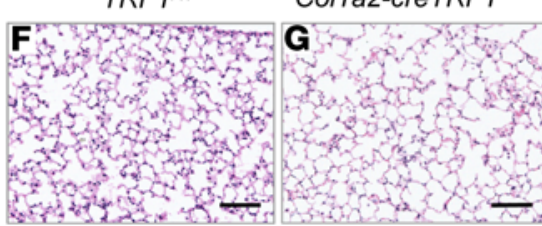

J $-T^{T F 1^{F / F}}$

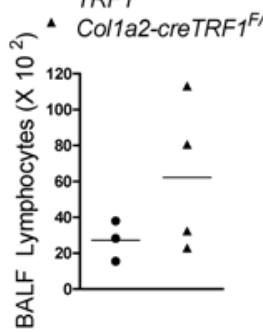

C - $T R F 1^{F / F}$

- Col1a2-creTRF1 ${ }^{F / F}$

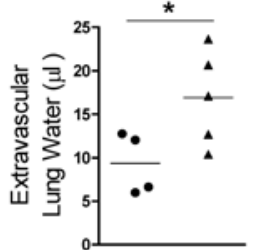

Col1a2-creTRF1F/F

.
stained lung sections harvested from TRF1 ${ }^{f / f l}$ and Col1a2-Cre TRF1 ${ }^{f l / f l}$ mice after 6 months of weekly tamoxifen injections. Scale bars: $100 \mu \mathrm{m}$. (H-J) Differential cell count of BALF obtained from TRFT ${ }^{f / / f l}$ and Col1a2-Cre $T R F T^{f l / f l}$ mice 2 days after tamoxifen injection. There was no significant difference in the numbers of macrophages, PMNs, and lymphocytes

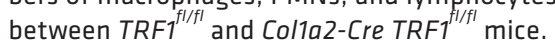
$n=3-4$ mice/group, $P>0.05$, 2-tailed Student's $t$-test.

Deletion of TRF1 in collagen-expressing cells causes pulmonary edema. To examine whether telomere dysfunction leading to lung fibrosis is unique to type II AECs or whether it could be the consequence of telomere dysfunction in other resident lung cells, we deleted TRF1 in cells expressing type 1 collagen by crossing $T R F 1^{f l / f l}$ mice with mice engineered to inducibly express Cre recombinase using the Collalpha2 promoter (Colla2-Cre/ERT) (21). Fibroblasts isolated from tamoxifen treated Colla2-Cre TRF1 ${ }^{\text {fllfl }}$ mice showed nearly 90\% reduction in TRF1 expression compared with $T R F 1^{f l f l}$ mice (Figure $4 \mathrm{~A}$ ). When tamoxifen was administered to Col1a2-Cre TRF1 $1^{f l f l}$ mice, $60 \%$ of these mice died by day 7 . In contrast, all tamoxifen-treated TRF$1^{f l f l}$ control mice survived (Figure $4 \mathrm{~B}$ ). To determine why mice died, the histopathology of major organs (lungs, kidney, skin, liver, and spleen) (Figure 4, D and E, and Supplemental Figure 2) was reviewed. The only abnormality identified was edema fluid in the alveolar spaces of Colla2-Cre TRF1 $1^{f / f l}$ mice (Figure 4, D and E). Gravimetric quantification of extravascular lung water (22) 48 hours after tamoxifen injection confirmed a more than 2-fold excess water in lungs of Col1a2-Cre TRF $1^{f / f l}$ mice (Figure 4C). BAL fluid analysis of Colla2-Cre TRF1 $1^{f l f l}$ mice 48 hours after tamoxifen injection revealed nonsignificant increases in macrophages, neutrophils, and lymphocytes in moribund Col1a2-Cre TRF1 fl/fl mice, indicating that the morbidity is independent of acute inflammation (Figure 4, H-J). Immunophenotyping of whole-lung homogenates of Colla2-Cre TRF $1^{f l / f}$ mice 48 hours after tamoxifen injection revealed no difference in the relative fractions of collagen-expressing cells, T cells, B cells, macrophages, and fibrocytes (Supplemental Table 2). Weekly tamoxifen treatment of $T R F 1^{f l / f l}$ control mice and the surviving Col1a2-Cre TRF1 $1^{f / f l}$ mice for up to 6 months did not result in lung remodeling or fibrosis (Figure 4, F and $\mathrm{G}$ ). These findings suggest that maintaining normal telomere function in lung mesenchymal cells is critical to preserving the lung barrier and that telomere dysfunction leading to lung fibrosis may be specific to type II AECs and not simply a consequence of telomere dysfunction within any resident lung cell.

Epithelial cell dysfunction and lung remodeling in SPC-Cre TRF1 fl/fl mice. Because telomere dysfunction leads to cellular senescence (23), we next sought to determine whether senescent cells are present in the lungs of SPC-Cre TRF1 $1^{f / f l}$ mice. To evaluate them for senescence, frozen lung sections were stained for senescence-associated $\beta$-galactosidase (SA- $\beta$-gal) and a population of SA- $\beta$-gal ${ }^{+}$cells (blue cells, Figure $5 \mathrm{~A}$ and Supplemental Figure 3) were identified in SPC-Cre TRF1 $1^{f / f l}$ mice but not in age-matched, tamoxifentreated, $T R F 1^{f / f l}$ control lungs. Furthermore, when lung digests of $S P C$-Cre $T R F 1^{f / / f}$ mice were stained for 
A

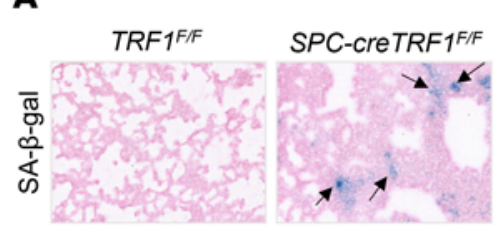

B
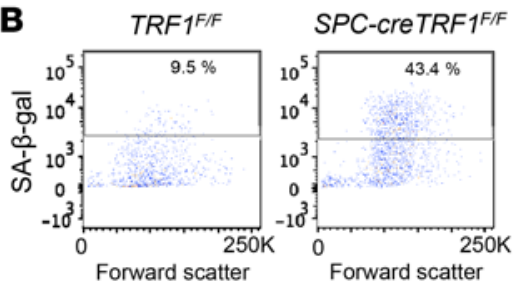

TRF1F/F

8 M Tamox

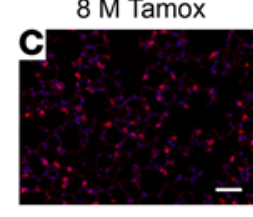

G

$\circ T R F 1^{F / F}$

$\triangle$ SPC-cre TRF ${ }^{\mathrm{F} / F}$

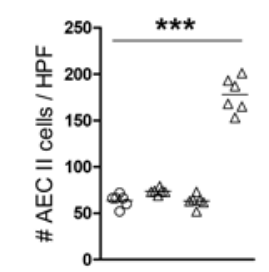

Tamox $8 \mathrm{M} 2 \mathrm{~W} 3 \mathrm{M} 8 \mathrm{M}$

\section{SPC-creTRF1FF}

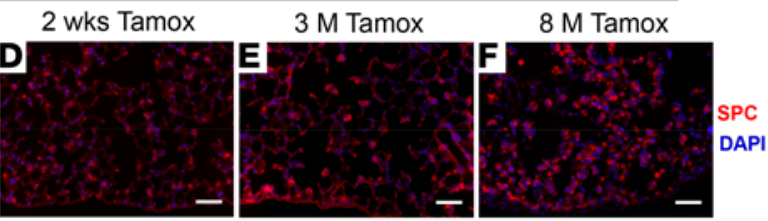

H

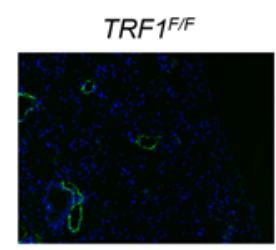

SPC-creTRF1F/F

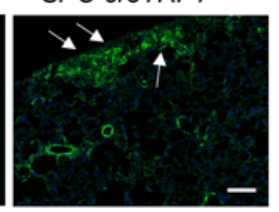

Figure 5. Telomere dysfunction in type II AECs causes cellular senescence and hyperplasia. (A) Senescenceassociated $\beta$-galactosidase (SA- $\beta$-gal) staining of sections of lung harvested from $T R F 7^{\text {fl/fl }}$ and SPC-Cre $T R F 7^{f l / f l}$ mice following 8 months of weekly injections of tamoxifen ( $250 \mathrm{mg} / \mathrm{kg}$ body weight). Note the blue SA- $\beta$-gal ${ }^{+}$cells (arrows) in lungs of SPC-Cre TRFfl/fl mice but not in $T R F 7^{f / f l}$ mice. (B) SA- $\beta$-gal activity was detected in $43.4 \%$ of type II AECs isolated from SPC-Cre TRF $7^{f l / f l}$ lungs compared with $9.5 \%$ of type II AECs isolated from TRFT ${ }^{f / f l}$ mice when analyzed by flow cytometry. (C-F) Immunostaining of SPC (red) in sections of lung harvested from $T R F 7^{f l / f l}$ and SPC-Cre $T R F^{f l / f l}$ mice treated with weekly tamoxifen injections for 2 weeks (D), 3 months (E) or 8 months (C and F). Note the increased density of type II AECs in SPC-Cre $T R F 7^{f / f l}$ lung sections at the 8-month time point. Nuclei were stained with DAPI (blue). Scale bars: $50 \mu \mathrm{m}$. (G) Quantification of type II AECs. $n=6$ mice/group, ${ }^{* *} P<0.0001$, 1-way ANOVA. (H) Immunostaining of $\alpha$-smooth muscle actin ( $\alpha$-SMA, green) in sections of lung harvested from $T R F 7^{f / f l}$ and SPC-Cre TRF $F^{f / / f l}$ mice treated with weekly tamoxifen injections for 8 months. $\alpha-S M A^{+}$cells (green) indicated by white arrows. DAPI (blue). Scale bar: $100 \mu \mathrm{m}$.

SA- $\beta$-gal and analyzed by flow cytometry, we found that $43.4 \%$ of Epcam ${ }^{+}$lung epithelial cells were SA- $\beta$ $\mathrm{gal}^{+}$(Figure 5B) compared with $9.5 \%$ in control lungs. These findings show that there are increased numbers of senescent type II AECs in SPC-Cre TRF1 $1^{f / f l}$ mice.

Type II AEC hyperplasia is a histopathologic finding in IPF lungs (3). To determine whether type II AEC hyperplasia is similarly found in SPC-Cre TRF1 fl/fl mice, the lungs of mice treated for 2 weeks, 3 months, or 8 months (Figure 5, D-F) with tamoxifen injections were immunostained for SPC and the density of type II AECs quantified. This analysis revealed no difference in the density of type II AECs at the 2-week and 3-month time points and a significant increase at the 8-month time point in SPC-Cre TRF1 fl/f mice compared with $T R F 1^{f l / f l}$ control lungs (Figure 5, C-G). An additional feature of IPF is increased numbers of $\alpha$-smooth muscle actin ( $\alpha$-SMA) mesenchymal cells (24). Examining the expression of $\alpha$-SMA in lung sections of $S P C$-Cre $T R F 1^{f l / f l}$ mice and $T R F 1^{f l f l}$ mice by immunofluorescence revealed foci of $\alpha$-SMAexpressing cells in $S P C$-Cre $T R F 1^{1 / f l}$ mice at the 8-month time point (Figure $5 \mathrm{H}$ ).

Telomere shortening in type II AECS of SPC-Cre TRF $1^{f / f t}$ mice. The telomeres are short in type II AECs of familial and sporadic IPF patients $(4,25)$. To determine whether telomeres in type II AECs of SPC-Cre $T R F 1^{f l f l}$ mice are also shortened, we measured telomere length by quantitative fluorescence in situ hybridization (Q-FISH). Quantification of telomere signal showed that the telomere signal intensity was similar in SPC-immunoreactive cells of SPC-Cre TRF1 $1^{f / f l}$ mice treated with tamoxifen for 3 months compared with $T R F 1^{f l / l}$ controls (Figure 6E). In contrast, it was 5-fold less intense in SPC-immunoreactive cells of $S P C$-Cre $T R F 1^{f l f l}$ mice 8 months after weekly tamoxifen administration compared with TRF1 $1^{f / f l}$ control mice (Figure 6, A-E). These findings show that long-term deletion of TRF1 leads to telomere shortening within type II AECs.

\section{Discussion}

Molecular defects in IPF type II AECs include short telomeres $(4,5)$ and expression of molecular markers of senescence $(6,26)$. The findings summarized above show that telomere dysfunction isolated to type II AECs leads to the development of lung remodeling and fibrosis. This lung remodeling evolves as the mice age and is not preceded by histopathologic evidence of lung injury or type II AEC depletion, although there was evidence for activation of a DNA damage response in these cells. Nevertheless, despite histopathologically normal lungs, SPC-Cre TRF1 $1^{f / f l}$ mice are more susceptible to influenza A infection, indicating that telomere 


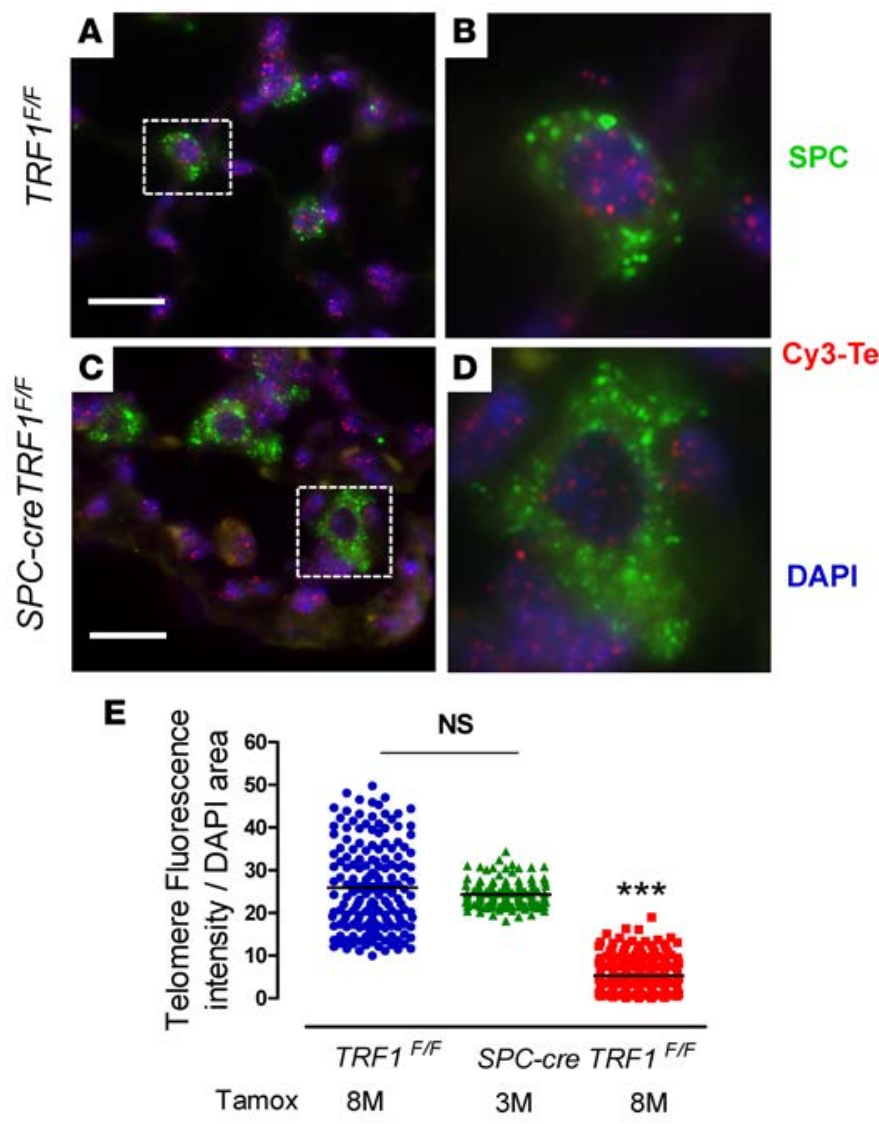

Figure 6. Measurement of telomere length by quantitative fluorescence in situ hybridization (Q-FISH). (A-D) Telomere length was measured by Q-FISH on sections of lung harvested from TRF1flfl and $S P C$-Cre TRF $7^{f l f f l}$ mice following 3 months and 8 months of weekly injections of tamoxifen ( $250 \mathrm{mg} / \mathrm{kg}$ body weight). Cy3-labeled telomere signal (red), surfactant protein C-immunoreactive cells (SPC, green), and nuclei were stained with DAPI (blue). Magnification: $\times 40$. Scale bars: $20 \mu \mathrm{m}$. Panels $\mathbf{B}$ and $\mathbf{D}$ are magnified images of boxed areas in $\mathbf{A}$ and $\mathbf{C}$, respectively. (E) Quantification of telomere fluorescence intensity with respect to DAPI area analyzed using Metamorph software. Each data point represents telomere fluorescence intensity in an SPC-positive cell. Data were collected from 5 mice/group, ${ }^{* *} P<0.0001$, 1-way ANOVA. NS, not significant.

dysfunction in type II AECs leads to a loss of alveolar epithelial cell function in maintaining lung homeostasis during times of stress. Telomere dysfunction within type II AECs may predispose the host to lung injury, worse outcomes following external stressors such as lung infection, or ultimately lung fibrosis.

Telomere uncapping leads to cellular senescence $(27,28)$. Consistent with these reports, our studies show increased numbers of senescent type II AECs in SPC-Cre TRF1 $1^{\text {flfl }}$ mice. Accumulation of senescent type II AECs contributes to the type II AEC hyperplasia observed in SPC-Cre TRF1 fl/fl mice at the 8-month time point. In addition, we observed senescent type II AEC accumulation in the areas of fibrosis, which was not evident at the 2-week or 3-month time point when no major lung remodeling was present. The accumulation of senescent cells correlated temporally to the development of lung fibrosis, suggesting that they may contribute to the fibrotic process.

Conditional deletion of shelterin proteins has enabled study of the consequences of telomere dysfunction in the skin (17), bone marrow (16), and lung $(18,23)$. These models have recapitulated features of telomere-mediated disease. For example, deletion of TRF1 from keratinocytes or hematopoietic cells leads to hyperkeratosis or bone marrow failure, respectively $(16,17)$. Within lung, we show that telomere dysfunction and shortening isolated to type II AECs leads to spontaneous lung remodeling and fibrosis. The lung fibrosis mediated by telomere dysfunction appears to be selective to type II AECs, as telomere dysfunction in collagen-expressing cells did not produce lung fibrosis. In contrast, induction of telomere dysfunction by uncapping telomeres in collagen-expressing cells caused pulmonary edema without evidence for pathological abnormalities in other tissues such as skin, liver, spleen, and kidney. These findings suggest that lung edema might have stemmed from dysfunctional lung fibroblasts or pericytes that are required to maintain the barrier in the lung. Overall, these findings suggest that the organ-specific manifestations of telomere dysfunction may be due to telomere dysfunction in a specific cell subtype within that organ. In the case of lung fibrosis, the relevant cell is the type II AEC.

Similar to our findings, a recent manuscript by Povedano et al. reported that selective deletion of TRF1 in type II AECs of SFTPC-Cre ${ }^{E R T 2}$ KFP $^{\text {lox-STOP-lox }}$ mice led to a DNA damage response ( $\gamma \mathrm{H} 2 \mathrm{AX}$ immunoreactive cells), increase in lung macrophages, and pulmonary fibrosis (18). However, unlike our SPC-Cre TRF $1^{f / f l}$ mice that have type II AEC hyperplasia and no histopathologic evidence of acute lung injury, type II AEC death, or apoptosis, Povedano et al. reported significant type II AECs cell death and lung injury following deletion of TRF1 in type II AECs of Trf1 10x/lox SFTPC-Cre ${ }^{E R T 2}$ KFPlox-STOP-lox mice (18). These discrepancies could possibly be explained by differences in the SFTPC Cre drivers, the dosing and timing of tamoxifen administration, which could lead to different levels of Cre expression, or coexpression of KFP in Trf $1^{\text {lox } / \text { lox } S F T P C-C r e e^{E R T 2}}$ KFP $P^{\text {lox-STOP-lox }}$ mice, which could be toxic to type II AECs in the context of uncapped telomeres. Furthermore, telomeres were not shortened in lung cells 8 weeks after tamoxifen administration in Trflox/lox SFTPC-Cre ${ }^{\text {ERT2 }}$ $K F P^{\text {lox-STOP-lox }}$ mice, similar to our finding of normal telomere length 12 weeks after initiating tamoxifen treatment. In contrast, we found telomeres were 5-fold shorter in type II AECs cells of SPC-Cre TRF1 $1^{f / f l}$ mice at the time of peak fibrosis and death of mice 8 months after initiating tamoxifen administration. Consistent with 
slow turnover of type II AECs, these data show that it takes up to 8 months to develop short telomeres in type II AECs following deletion of TRF1. Collectively, our findings show that type II AEC telomere dysfunction and shortening, in the absence of lung injury, may be sufficient to cause lung fibrosis.

Histopathologic evidence of lung fibrosis in SPC-Cre TRF1 $1^{f / f l}$ mice was identified months after deletion of TRF1, which led to the uncapping of telomeres and activation of a DNA damage response. This finding is consistent with reports that pulmonary fibrosis in patients with TERT or TERC mutations typically develops at age 50 or greater, despite carrying the mutation from birth, and having short telomeres since childhood (29). Why there is a relative delay in developing fibrosis in patients or our model remains unresolved. One possibility is that although senescent type II AECs are detectable at the time of uncapping telomeres, they may need to accumulate to a critical mass such that the profibrotic mediators they release have the highest activity. Another possibility is that senescence reprogramming of cells alters their phenotype over time (30) and that it takes time for the senescent cells to develop a profibrotic phenotype. A third possibility is that the profibrotic phenotype of type II AECs is related to critically short telomeres, which requires more than 3 months to develop in SPC-Cre TRF1 $1^{f / f l}$ mice. Finally, although the SPC-Cre TRF1 fl/fl mice were housed in a barrier facility, it is possible that unrecognized environmental exposures interact with telomere shortening in type II AECs to cause lung fibrosis. Which of these (or other) mechanisms are at play in this model or IPF will require additional studies to resolve.

$S P C$-Cre TRF1 $1^{f l f l}$ mice provide a potentially novel in vivo model of lung remodeling and fibrosis. The SPC-Cre TRF ${ }^{f l / f l}$ mouse model of fibrosis recapitulates many pathologic findings in IPF including short telomeres in type II AECs $(4,25)$, accumulation of senescent type II AECs $(6,26)$, type II AEC hyperplasia (3), increased burden of alveolar macrophages (25), accumulation of $\alpha$-SMA-immunoreactive mesenchymal cells $(24,31)$, elevated levels of active TGF- $\beta 1$ (32), and lung fibrosis (3). In addition to recapitulating features of IPF, the SPC-Cre TRF ${ }^{f l f l}$ mouse model of fibrosis has advantages over the commonly used bleomycin model of lung fibrosis. Most notable are that fibrosis occurs spontaneously and progresses to death of all mice over months, a time interval more consistent with fibrosis progression in IPF patients. In contrast, the lung fibrosis in the bleomycin model is not progressive and is mediated by acute lung injury induced by intra-alveolar instillation of a chemotherapeutic compound. Although SPC-Cre TRF $1^{f l f l}$ mice do not manifest all pathologic features of IPF (e.g., fibroblast foci were not identified) the findings in SPC-Cre TRF $1^{f l f l}$ mice suggest that IPF may be mediated by telomere dysfunction in type II AECs and that the progressive terminal course of IPF is due to irreversible telomere shortening in type II AECs. SPC-Cre TRF fllfl mice should be useful to study this possibility and identify key cellular and molecular mechanisms of lung fibrosis mediated by telomere dysfunction in IPF type II AECs.

\section{Methods}

Antibodies and reagents. Commercially available antibodies were purchased from the following vendors. Anti- $\gamma$ H2AX-phosphorylated (Ser 139) (Biolegend, catalog 613402), TRF1 (Cell Signaling Technology, catalog 3529), biotinylated rat anti-mouse CD45 (BD Biosciences, catalog 553078), rabbit antimouse SPC (EMD Millipore, catalog ab3786), smooth muscle actin (Dako, catalog 0851), monoclonal anti-mouse CD326 (Epcam) APC (eBioscience, catalog 17-5791), rabbit anti-mouse $\beta$-actin (Abcam, catalog ab8227), goat anti-rabbit IgG-HRP (Santa Cruz Biotechnology, catalog sc-2004), and goat antimouse IgG-HRP (Santa Cruz Biotechnology, catalog sc-2005). The following fluorescence-conjugated primary antibodies used for immunophenotyping were acquired from Biolegend. PerCP/Cy5.5 antimouse CD45 (catalog 103131), PE anti-mouse CD184 (catalog 146505), PE/Cy7 anti-mouse CD3 (catalog 100219), APC anti-mouse IL-21R (catalog 131909), APC/Cy7 anti-mouse CD16/32 (catalog 101327), Brilliant Violet 421 anti-mouse CD206 (catalog 141717), and Brilliant Violet 421 anti-mouse F4/80 (catalog 123131). Live/Dead Fixable Aqua was purchased from Thermo Fisher Scientific (cata$\log$ L34965). Fluorescent secondary antibodies goat anti-mouse Alexa Fluor 488 (catalog A11001), goat anti-rabbit Texas Red (catalog T6391), and donkey anti-goat Alexa Fluor 546 (catalog A11056) were purchased from Life Technologies. Cell Viability Dye eFluor 780 was purchased from eBioscience (catalog 65-0865-14). For hydroxyproline assays, all reagents were purchased from Sigma-Aldrich.

Tamoxifen administration. Tamoxifen (Sigma-Aldrich, catalog T5648, suspended in peanut oil) was injected $\left(250 \mathrm{mg} / \mathrm{kg}\right.$ body weight once per week) intraperitoneally to SPC-Cre TRF $1^{f / f l}$ mice, Colla2 CreTRF1 $1^{f l f l}$ mice, and TRF1 $1^{f l f l}$ littermate controls beginning at 8 weeks of age. 
Hydroxyproline assay. Hydroxyproline levels were quantified as described previously (33). Murine lungs were homogenized in $1 \mathrm{ml}$ water. One hundred and twenty-five microliters of 50\% trichloroacetic acid was added and the suspension incubated on ice for 20 minutes. Samples were centrifuged at $800 \mathrm{~g}$ for 5 minutes at $4^{\circ} \mathrm{C}$. The supernatant was discarded and $1 \mathrm{ml}$ of $12 \mathrm{~N} \mathrm{HCl}$ added to the pellet, which was then baked in an oven at $110^{\circ} \mathrm{C}$ for 24 hours. Dried pellets were reconstituted in $2 \mathrm{ml}$ distilled water. Sample or serially diluted 6-hydroxyproline standard $(200 \mu \mathrm{l})$ was added to chloramine $\mathrm{T}(1.4 \%$ chloramine $\mathrm{T}$ in $0.5 \mathrm{M} \mathrm{Na}$ acetate and $10 \%$ isopropanol) and incubated at room temperature for 20 minutes. Five hundred microliters of Ehrlich's solution (1 M p-dimethylaminobenzaldehyde [ $p$-DMBA] in 70\% isopropanol and 30\% perchloric acid] was added and the mixture incubated at $65^{\circ} \mathrm{C}$ for 15 minutes and absorbance measured on a spectrophotometer at $550 \mathrm{~nm}$.

Histopathology and immunofluorescence staining. Lungs were perfused with $10 \%$ formalin to an inflation pressure of $20 \mathrm{~cm} \mathrm{H}_{2} \mathrm{O}$ and fixed overnight before embedding in paraffin and sectioning at 4- $\mu \mathrm{m}$ thickness. For histopathology, tissues were stained with H\&E or Masson's trichrome. For immunohistochemistry, tissues were deparaffinized in xylene, rehydrated in an ethanol gradient, permeabilized with $0.1 \%$ Triton X-100, and antigen retrieved by heating at $95^{\circ} \mathrm{C}$ for 20 minutes in $10 \mathrm{mM}$ sodium citrate buffer, $\mathrm{pH}$ 6.0. Sections were blocked (3\% BSA, 10\% goat serum, PBS) and incubated with primary antibodies (SPC 1:300 dilution, SMA 1:75 dilution, and $\gamma \mathrm{H} 2 \mathrm{AX}$ 1:500 dilution) overnight at $4^{\circ} \mathrm{C}$. Tissues were washed thrice and incubated with appropriate secondary antibodies at $20^{\circ} \mathrm{C}$, washed, and mounted using Prolong Gold Antifade Mounting Medium with DAPI (Life Technologies). Images were acquired on a Leica confocal microscope.

Telomere Q-FISH assay. Telomere lengths were measured on paraffin-embedded tissue sections by Q-FISH (34). Briefly, after deparaffinization, tissues underwent antigen retrieval by heating in a microwave in $10 \mathrm{mM}$ sodium citrate buffer, $\mathrm{pH}$ 6.5, then incubating for 15 minutes in $0.01 \mathrm{M} \mathrm{HCL}$ containing $1 \%$ pepsin (Thermo Fisher Scientific). The tissues were washed and air-dried before treating with $10 \mathrm{mg} / \mathrm{ml}$ RNase solution (QIAGEN). After washing again, the tissues were incubated with $0.3 \mu \mathrm{g} / \mathrm{ml}$ PNA FISH probe TelC-Cy3 (Panagene) suspended in 70\% formamide, 30\% water, and $10 \mathrm{mM}$ Tris, $\mathrm{pH} 7.5$, heated at $78^{\circ} \mathrm{C}$ for 10 minutes, and then left overnight at room temperature. The tissues were then washed sequentially with formamide buffer and then PBS containing $0.1 \%$ Tween, blocked with 3\% BSA (Sigma-Aldrich) and $10 \%$ donkey serum, and incubated overnight at $4^{\circ} \mathrm{C}$ with goat anti-SPC antibody. Tissues were washed with PBS containing $0.1 \%$ Tween and incubated with donkey anti-goat Alexa 488 secondary antibody at $20^{\circ} \mathrm{C}$ for 1 hour, washed, and mounted using Prolong Gold Antifade Mounting Medium with DAPI. Images were acquired using a Zeiss Axio Imager 2 microscope and telomere signal intensity quantified using MetaMorph imaging analysis software (Molecular Devices).

Immunophenotyping by flow cytometry. Murine lungs were digested in $10 \mathrm{mg} / \mathrm{ml}$ Dispase (Life Technologies, catalog 17105-041) for 1 hour and suspended in DMEM containing 10\% FBS and DNase I. Cell suspensions were serially filtered through $70-\mu \mathrm{m}$ and $40-\mu \mathrm{m}$ filters. Fluorescence-conjugated antibodies were incubated in blocking buffer containing $1 \%$ BSA for 30 minutes at $4^{\circ} \mathrm{C}$. In some groups, cells were fixed and permeabilized with Cytofix/Cytoperm according to the manufacturer's protocol (BD Biosciences). Cells were then stained with fluorescein-conjugated rabbit anti-collagen type I antibody (Rockland Immunochemicals) in Cytoperm wash buffer for 30 minutes at $4^{\circ} \mathrm{C}$. After washing, cells were suspended in buffer containing 1\% BSA and analyzed on an LSR II flow cytometer (BD Biosciences) and acquired data were analyzed using the integrated FlowJO software v10.

BAL fluid collection. BAL fluid was collected from euthanized mice by lavaging lungs with $1 \mathrm{ml}$ PBS. Recovered BAL fluid was centrifuged at $800 \mathrm{~g}$ for 5 minutes at $4^{\circ} \mathrm{C}$ and the supernatant recovered for analysis. Red blood cells were lysed in the cell pellet with ammonium chloride-potassium (ACK) buffer and the cells recentrifuged and then suspended in $1 \mathrm{ml}$ PBS. The total numbers of cells were counted using a hemocytometer. Cells were cytospun and cell differentials determined by modified Giemsa stain (Electron Microscopy Sciences).

Enzyme-linked immunosorbent assay. BAL fluid supernatant was used to determine active TGF- $\beta 1$ levels according to the manufacturer's instructions (Biolegend, catalog 437707).

Isolation of murine AECs. Lungs from tamoxifen-treated SPC-Cre TRF1 $1^{f / f l}$ mice and age-matched $T R F 1^{f l / f l}$ controls were serially lavaged with PBS and then perfused via the pulmonary artery with PBS containing $0.5 \%$ EDTA. Lungs were digested in $10 \mathrm{mg} / \mathrm{ml}$ Dispase suspended in DMEM containing $10 \%$ FBS for 1 hour and then minced in the presence of DNase I. The suspended cells were serially 
filtered, and then negatively selected for CD45 (CELLection Biotin Binder Kit) using magnetic beads bound to anti-CD45 antibody. The cell suspension was stained with Pacific Blue-conjugated primary antibody against CD45 (Life Technologies, catalog MCD 4528), APC-Epcam (eBioscience), and Cell Viability Dye eFluor 780. After a final wash, cells were suspended in PBS, analyzed by flow cytometry, and sorted on a BD Aria cell sorter for Epcam-positive cells.

$S A$ - $\beta$-gal assay. To measure SA- $\beta$-gal (35), perfused lungs were processed to obtain cellular suspensions followed by depletion of CD45-positive cells (CELLection Biotin Binder Kit) using a biotinylated CD45 antibody. The CD45-depleted cell suspension was pretreated with bafilomycin A1 followed by treatment with 5 -dodecanoylaminofluorescein di- $\beta$-D-galactopyranoside (C12FDG). Cells were then incubated with Pacific Blue-conjugated rat anti-mouse CD45, APC-Epcam, and Cell Viability Dye eFluor 780, washed, and analyzed by flow cytometry with FlowJO software (Version X). To visualize senescent cells by cytochemistry, 5-bromo-4-chloro-3-indolyl- $\beta$-D-galactoside (X-gal) was used as a substrate on lung cryosections (36).

Extravascular lung water measurement (EVLW). EVLW was measured by gravimetry as described (22). Mouse lungs were harvested from the thoracic cavity and homogenized after adding a fixed quantity of water. Lung homogenate was weighed immediately and then dried overnight at $65^{\circ} \mathrm{C}$ in an oven to record the dry weight. Hemoglobin in the lung homogenate and in the blood was measured to calculate the weight of blood in the lungs. The difference between blood water and lung water provided the EVLW value.

Isolation of pulmonary fibroblasts. Pulmonary fibroblasts were isolated from Colla2-Cre TRF $1^{f l / f l}$ mice and $T R F 1^{f / f l}$ mice by digesting lungs in $10 \mathrm{mg} / \mathrm{ml}$ Dispase suspended in DMEM containing 10\% FBS for 1 hour at $37^{\circ} \mathrm{C}$, and then minced in the presence of DNase I. The cell lysate was centrifuged at $800 \mathrm{~g}$ for 10 minutes at $4^{\circ} \mathrm{C}$. The cell pellet was resuspended in DMEM medium containing $10 \%$ FBS and cultured for 4 days. Nonadherent cells were rinsed and adherent fibroblasts were cultured for 3 more days prior to harvest for analysis.

Influenza virus infection. Anesthetized mice were inoculated intranasally with 5,000 PFU of purified human influenza A/PR/8/34 (H1N1) virus (Advanced Biotechnologies, catalog 10-210-500) suspended in $25 \mu 1$ sterile PBS. The mice were recovered and monitored 3 times daily and their survival recorded. Moribund mice were euthanized by $\mathrm{CO}_{2}$ inhalation and cervical dislocation.

Preparation of cell lysates and immunoblotting. Cells were washed with ice-cold PBS and then lysed in RIPA buffer (50 mM Tris- $\mathrm{HCl}$ [pH 7.4], $150 \mathrm{mM} \mathrm{NaCl}$, Nonidet P-40, $0.25 \%$ sodium deoxycholate, 1 mM EDTA, $1 \mathrm{mM}$ phenylmethylsulfonylfluoride, $1 \mathrm{mM}$ sodium orthovanadate, $1 \mathrm{mM}$ sodium fluoride) containing protease and phosphatase inhibitor cocktail (Sigma-Aldrich). Protein concentration was measured in the lysate using a Pierce BCA protein assay kit (Thermo Fisher Scientific). Equal volumes of sample supernatants were adjusted to $1 \mathrm{mg} / \mathrm{ml}$ protein, dissolved in $4 \times$ SDS sample buffer, and denatured by boiling for 5 minutes. Sample proteins were resolved in 4\%-15\% SDS-PAGE gradient gels (Bio-Rad), transferred onto nitrocellulose membranes, blocked in TBS buffer (10 mM Tris- $\mathrm{HCl}, 150 \mathrm{mM} \mathrm{NaCl}, \mathrm{pH}$ 7.4) containing 5\% milk and incubated with primary (TRF1 diluted $1: 1,000 ; \beta$-actin diluted 1:2,500) and secondary (goat anti-mouse IgG-HRP) antibodies. The proteins were detected by an ECL kit (Thermo Fisher Scientific), scanned, and analyzed by ImageJ software (NIH).

Statistics. Statistical analysis was performed using 2-tailed Student's $t$ test. Welch's correction was applied for analysis of unpaired groups. Differences in survival were analyzed by log-rank test. Analysis between multiple groups was performed by 1 -way ANOVA with Tukey's post-hoc test. $P$ values less than 0.05 were considered significant. Data are represented as the mean \pm SEM.

Study approval. TRF ${ }^{f l / l}$ mice were purchased from Jackson Laboratories, Colla2-Cre/ERT mice were provided by Steve Nishimura (UCSF), and SPC-Cre ER ${ }^{T 2}$ rtTA mice were provided by Hal Chapman (UCSF). Mice were bred and housed in pathogen-free conditions in accordance with the guidelines of Laboratory Animal Resource Center (LARC). All animal procedures were carried out using protocols approved by the Institutional Animal Care and Use Committee, UCSF.

\section{Author contributions}

RPN designed, performed and analyzed experiments and wrote the manuscript. SD, JLL, and GG performed experiments. BM and MD designed and performed experiments. MRL and JRR designed experiments and provided critical comments on the manuscript. PJW conceived, designed and supervised the study. 


\section{Acknowledgments}

This work was funded by The Harroun Family Foundation and the Nina Ireland Program in Lung Health. The coauthors thank Rachel Madding for technical assistance.

Address correspondence to: Paul J. Wolters, University of California, San Francisco, Box 0111, San Francisco, California 94143-0111, USA. Phone: 415.514.2601; E-mail: paul.wolters@ucsf.edu.

1. Raghu G, Weycker D, Edelsberg J, Bradford WZ, Oster G. Incidence and prevalence of idiopathic pulmonary fibrosis. Am J Respir Crit Care Med. 2006;174(7):810-816.

2. Armanios M. Telomeres and age-related disease: how telomere biology informs clinical paradigms. J Clin Invest. 2013;123(3):996-1002.

3. Wolters PJ, Collard HR, Jones KD. Pathogenesis of idiopathic pulmonary fibrosis. Annu Rev Pathol. 2014;9:157-179.

4. Alder JK, et al. Short telomeres are a risk factor for idiopathic pulmonary fibrosis. Proc Natl Acad Sci US A. 2008;105(35):13051-13056.

5. Kropski JA, et al. Extensive phenotyping of individuals at risk for familial interstitial pneumonia reveals clues to the pathogenesis of interstitial lung disease. Am J Respir Crit Care Med. 2015;191(4):417-426.

6. Disayabutr S, et al. miR-34 miRNAs regulate cellular senescence in type II alveolar epithelial cells of patients with idiopathic pulmonary fibrosis. PLoS One. 2016;11(6):e0158367.

7. Stuart BD, et al. Effect of telomere length on survival in patients with idiopathic pulmonary fibrosis: an observational cohort study with independent validation. Lancet Respir Med. 2014;2(7):557-565.

8. Fingerlin TE, et al. Genome-wide association study identifies multiple susceptibility loci for pulmonary fibrosis. Nat Genet. 2013;45(6):613-620.

9. Armanios MY, et al. Telomerase mutations in families with idiopathic pulmonary fibrosis. N Engl J Med. 2007;356(13):1317-1326.

10. Tsakiri KD, et al. Adult-onset pulmonary fibrosis caused by mutations in telomerase. Proc Natl Acad Sci US A. 2007;104(18):7552-7557.

11. Cronkhite JT, et al. Telomere shortening in familial and sporadic pulmonary fibrosis. Am J Respir Crit Care Med. 2008;178(7):729-737.

12. Safa WF, Lestringant GG, Frossard PM. X-linked dyskeratosis congenita: restrictive pulmonary disease and a novel mutation. Thorax. 2001;56(11):891-894.

13. Utz JP, Ryu JH, Myers JL, Michels VV. Usual interstitial pneumonia complicating dyskeratosis congenita. Mayo Clin Proc. 2005;80(6):817-821.

14. de Lange T. Shelterin: the protein complex that shapes and safeguards human telomeres. Genes Dev. 2005;19(18):2100-2110.

15. Calado RT, Dumitriu B. Telomere dynamics in mice and humans. Semin Hematol. 2013;50(2):165-174.

16. Beier F, Foronda M, Martinez P, Blasco MA. Conditional TRF1 knockout in the hematopoietic compartment leads to bone marrow failure and recapitulates clinical features of dyskeratosis congenita. Blood. 2012;120(15):2990-3000.

17. Martínez P, et al. Increased telomere fragility and fusions resulting from TRF1 deficiency lead to degenerative pathologies and increased cancer in mice. Genes Dev. 2009;23(17):2060-2075.

18. Povedano JM, Martinez P, Flores JM, Mulero F, Blasco MA. Mice with pulmonary fibrosis driven by telomere dysfunction. Cell Rep. 2015;12(2):286-299.

19. Chapman HA, et al. Integrin $\alpha 6 \beta 4$ identifies an adult distal lung epithelial population with regenerative potential in mice. $J$ Clin Invest. 2011;121(7):2855-2862.

20. Hübner RH, et al. Standardized quantification of pulmonary fibrosis in histological samples. BioTechniques. 2008;44(4):507-517.

21. Zheng B, Zhang Z, Black CM, de Crombrugghe B, Denton CP. Ligand-dependent genetic recombination in fibroblasts : a poten tially powerful technique for investigating gene function in fibrosis. Am J Pathol. 2002;160(5):1609-1617.

22. Looney MR, Su X, Van Ziffle JA, Lowell CA, Matthay MA. Neutrophils and their Fc gamma receptors are essential in a mouse model of transfusion-related acute lung injury. J Clin Invest. 2006;116(6):1615-1623.

23. Alder JK, et al. Telomere dysfunction causes alveolar stem cell failure. Proc Natl Acad Sci U S A. 2015;112(16):5099-5104.

24. Ohta K, Mortenson RL, Clark RA, Hirose N, King TE. Immunohistochemical identification and characterization of smooth muscle-like cells in idiopathic pulmonary fibrosis. Am J Respir Crit Care Med. 1995;152(5 Pt 1):1659-1665.

25. Kropski JA, et al. Extensive phenotyping of individuals at risk for familial interstitial pneumonia reveals clues to the pathogenesis of interstitial lung disease. Am J Respir Crit Care Med. 2015;191(4):417-426.

26. Minagawa S, et al. Accelerated epithelial cell senescence in IPF and the inhibitory role of SIRT6 in TGF- $\beta$-induced senescence of human bronchial epithelial cells. Am J Physiol Lung Cell Mol Physiol. 2011;300(3):L391-L401.

27. Karlseder J, Broccoli D, Dai Y, Hardy S, de Lange T. p53- and ATM-dependent apoptosis induced by telomeres lacking TRF2 Science. 1999;283(5406):1321-1325.

28. Smogorzewska A, de Lange T. Different telomere damage signaling pathways in human and mouse cells. EMBO J. 2002;21(16):4338-4348.

29. Diaz de Leon A, et al. Telomere lengths, pulmonary fibrosis and telomerase (TERT) mutations. PLoS One. 2010;5(5):e10680

30. Ivanov A, et al. Lysosome-mediated processing of chromatin in senescence. J Cell Biol. 2013;202(1):129-143.

31. Marmai C, et al. Alveolar epithelial cells express mesenchymal proteins in patients with idiopathic pulmonary fibrosis. $A m J$ Physiol Lung Cell Mol Physiol. 2011;301(1):L71-L78.

32. Khalil N, O'Connor RN, Flanders KC, Unruh H. TGF-beta 1, but not TGF-beta 2 or TGF-beta 3, is differentially present in epithelial cells of advanced pulmonary fibrosis: an immunohistochemical study. Am J Respir Cell Mol Biol. 1996;14(2):131-138

33. Munger JS, et al. The integrin alpha $\mathrm{v}$ beta 6 binds and activates latent TGF beta 1: a mechanism for regulating pulmonary inflammation and fibrosis. Cell. 1999;96(3):319-328. 
34. Ricciardi M, Krampera M, Chilosi M. Quantitative fluorescence in situ hybridization on paraffin embedded tissue. Methods Mol Biol. 2013;976:167-173.

35. Debacq-Chainiaux F, Erusalimsky JD, Campisi J, Toussaint O. Protocols to detect senescence-associated beta-galactosidase (SA-betagal) activity, a biomarker of senescent cells in culture and in vivo. Nat Protoc. 2009;4(12):1798-1806.

36. Dimri GP, et al. A biomarker that identifies senescent human cells in culture and in aging skin in vivo. Proc Natl Acad Sci U S A. 1995;92(20):9363-9367. 